Tropical Journal of Pharmaceutical Research March 2019; 18 (3): 499-504

ISSN: 1596-5996 (print); 1596-9827 (electronic)

(C) Pharmacotherapy Group, Faculty of Pharmacy, University of Benin, Benin City, 300001 Nigeria.

\title{
Hh pathway expression in the blood, synovial cells and chondrocytes of different rheumatoid arthritis models
}

\author{
Yingyi Wu, Guangxia Yang, Jing Fei, Yang Huang* \\ Department of Rheumatology, Affiliated Hospital of Jiangnan University (Wuxi 4th People's Hospital), No. 200 Huihe Road, \\ Wuxi, Jiangsu 214062, China
}

*For correspondence: Email: YangHuangqaz@163.com; Tel: +86-51088082303

\begin{abstract}
Purpose: To investigate the effect of the hedgehog (Hh) pathway inhibitor, cyclopamine, and activator purmorphamine on articular cartilage cell proliferation.

Methods: Rats were subjected to AA and CIA models. Secondary paw swelling was measured at 12, $15,18,21,24,27$, and 30 days. The rats were sacrificed on day 30 . Tissues from the cartilage and knee joints were collected. Cell proliferation was determined by 3-(4,5-dimethylthiazol-2-yl)-2,5diphenyltetrazolium bromide (MTT) assay while cell apoptosis was determined by annexin V-fluorescein isothiocyanate/propidium iodide assay. Protein expression levels of Shh, Ptch1 and Gli1 were determined by Western blotting.

Results: Compared with the control group, arthritis index and secondary foot swelling of the adjuvant arthritis $(A A)$ and collagen-induced arthritis $(C / A)$ groups deteriorated significantly $(p<0.05)$. MTT data revealed that cyclopamine promoted articular cartilage cell proliferation of the $A A$ and CIA groups. The cell proliferation rates of $A A$ and CIA groups were significantly higher than that of control group $(p<$ $0.05)$. Flow cytometry showed that the cell apoptosis rates of $A A$ and $C I A$ groups were significantly lower than that of control group $(p<0.05)$. Compared with control, cyclopamine decreased the protein expression levels of sonic Hh, patched homologue 1 and glioma-associated oncogene homologue, but the effect of purmorphamine was the reverse.

Conclusion: Hh pathway inhibitor (cyclopamine) and activator (purmorphamine) affect the expression of Hh pathway. Disruption of the Hh pathway may be of potential therapeutic significance in protecting articular cartilage from rheumatoid arthritis.
\end{abstract}

Keywords: Adjuvant arthritis, Cyclopamine, Purmorphamine, Synovial cells, Chondrocytes, Hedgehog signaling pathway

\begin{abstract}
This is an Open Access article that uses a funding model which does not charge readers or their institutions for access and distributed under the terms of the Creative Commons Attribution License (http://creativecommons.org/licenses/by/4.0) and the Budapest Open Access Initiative (http://www.budapestopenaccessinitiative.org/read), which permit unrestricted use, distribution, and reproduction in any medium, provided the original work is properly credited.
\end{abstract}

Tropical Journal of Pharmaceutical Research is indexed by Science Citation Index (SciSearch), Scopus, International Pharmaceutical Abstract, Chemical Abstracts, Embase, Index Copernicus, EBSCO, African Index Medicus, JournalSeek, Journal Citation Reports/Science Edition, Directory of Open Access Journals (DOAJ), African Journal Online, Bioline International, Open-J-Gate and Pharmacy Abstracts

\section{INTRODUCTION}

Rheumatoid arthritis (RA) is an autoimmune disease leading to the symptoms like synovial inflammation, bone erosion, and cartilage destruction [1]. Almost all RA patients eventually develop varying degrees of joint dysfunction that poses great threat to human health [2]. Globally, the incidence of RA is approximately $1 \%$. Although the pathogenesis of RA is largely unknown, it is recognized that synoviocyte proliferation and inflammatory cell infiltration 
cause destruction of subsynovial cartilage and bone tissue [3].

Increasing evidence demonstrated that hedgehog $(\mathrm{Hh})$ signaling regulates cartilage cell growth and differentiation, and is very important for maintaining the biological function of cartilage cells $[4,5]$. The $\mathrm{Hh}$ protein family consists of sonic $\mathrm{Hh}$ (Shh), glioma-associated oncogene homologue (Gli1) and patched homologue 1 (Ptch1). The expression of Gli1 and Ptch1 are regarded as markers of Hh pathway activity [6].

The pathological role of $\mathrm{Hh}$ signaling in chondrocyte proliferation and differentiation as well as bone formation and metabolism have been verified in osteoarthritis (OA) [7-9]. Activation of the $\mathrm{Hh}$ signaling pathway was evident in the articular chondrocytes of patients with $\mathrm{OA}$, and inhibition of the Hh pathway by a specific blocker or gene silencing could significantly alleviate the condition of patients with OA $[10,11]$. These findings provide a new idea for the study and prevention of osteoarticular diseases. Some studies have shown that progressive cartilage destruction is a main feature of RA and OA. However, no study has reported the specific mechanism of the $\mathrm{Hh}$ signal pathway in RA. The effect of Hh signaling on synovial cells and articular chondrocytes in different rat models of RA in vitro has not been investigated.

The present study investigated the expression of the Hh signal pathway in the blood, synovial cells, and chondrocytes of an adjuvant arthritis (AA) rat model and a collagen-induced arthritis (CIA) rat model. The effects of $\mathrm{Hh}$ pathway inhibition or activation on the activation of synovial cells and chondrocytes in AA rats and CIA rats were observed to provide a new experimental basis for RA.

\section{EXPERIMENTAL}

\section{Experimental animals}

Sprague-Dawley (SD) rats weighing 220 - $250 \mathrm{~g}$ were purchased from Changzhou Cavens Experimental Animal Company, China. The rats were fed under standard laboratory conditions. In addition, the housing conditions of mice were controlled at a temperature of $22 \pm 2{ }^{\circ} \mathrm{C}$, a relative humidity of $55 \pm 5 \%$, and a 12-h light/12$\mathrm{h}$ dark cycle. Standard chow and water were given in the laboratory. The use of animals in the laboratory was conducted in accordance with international guidelines [12]. In addition, the animal experimental protocols designed in this study were approved by the University Ethics
Committee on Animal Research (approval no. LL170607Z). The SD rats were randomly divided into three groups as follows: Control, AA group, and CIA group. AA and CIA models were created as previously described $[13,14]$.

\section{Preparation of peripheral blood mononuclear cells (PBMCs)}

Fresh whole-blood samples of SD rats were used to isolate PBMCs, which were purified by density centrifugation using Lymphoprep (Gunma, Japan) according to the manufacturer's instructions. The PBMCs were cultured in cell culture medium (RPMI 1640) supplemented with $10 \%$ heat-inactivated (HI)-fetal bovine serum (FBS). Next, the PBMCs were activated with 10 $\mu \mathrm{g} / \mathrm{mL}$ lipopolysaccharide.

\section{Isolation and culture of synoviocytes and chondrocytes}

The rat tissues were obtained under aseptic conditions, were washed with D-Hanks solution (without $\mathrm{Ca}^{2+}$ and $\mathrm{Mg}^{2+}$ ), and then were cut into 1-mm pieces. Synoviocytes and chondrocytes were isolated with $5 \mathrm{mg} / \mathrm{mL}$ of pronase (Tokyo, Japan) at $37^{\circ} \mathrm{C}$ for $1 \mathrm{~h}$, followed by $2 \mathrm{mg} / \mathrm{mL}$ of collagenase (MO, USA) at $37^{\circ} \mathrm{C}$ for $6 \mathrm{~h}$ in cell culture medium (Kyoto, Japan) with $10 \%$ HI-FBS (Tulare, CA, USA) in a humidified atmosphere of $5 \% \mathrm{CO}_{2}$ and $95 \%$ air. To remove non adherent lymphoid cells, the cell culture medium was changed every 3 days. At confluence, the chondrocytes and synoviocytes were separated and passaged once. Next, they were seeded at high density and allowed to grow in cell culture medium as previously described [15-17].

\section{Drug treatment}

The treatment groups were as follows: blank control group, AA model negative control group, CIA model negative control group, AA model + cyclopamine group $(0.5,5$, and $20 \mu \mathrm{mol} / \mathrm{L})$, ClA model + cyclopamine groups $(0.5,5$, and 20 $\mu \mathrm{mol} / \mathrm{L}), \mathrm{AA}$ model + purmorphamine $(0.15,1.5$, and $6 \mu \mathrm{mol} / \mathrm{L})$, and $\mathrm{ClA}$ model + purmorphamine $(0.15,1.5$, and $6 \mu \mathrm{mol} / \mathrm{L})$.

\section{MTT assay}

Synoviocytes and chondrocytes in the logarithmic phase were collected, and $10 \times 10^{4}$ cells were added to each well in 96-well plates. Next, $50 \mu \mathrm{L}$ of cyclopamine $(0.5,5$, and 20 $\mu \mathrm{mol} / \mathrm{L})$ or purmorphamine $(0.15,1.5$, and 6 $\mu \mathrm{mol} / \mathrm{L}$ ) was added to the AA and CIA groups. In addition, $50 \mu \mathrm{L}$ of nutrient fluid was applied to each well. The 96-well plate was maintained at 
$37^{\circ} \mathrm{C}$ for $48 \mathrm{~h}$ with $5 \% \mathrm{CO}_{2}$ air. Thereafter, each well was added with $20 \mu \mathrm{L}$ of MTT. After $4 \mathrm{~h}$, the reaction was stopped by acidified DMSO. The optical density value (OD) at $490 \mathrm{~nm}$ of each well was measured by MK3 Enzyme Sign (Dutch Thunder Suddenly Company). In addition, cell proliferation was measured as described previously [15].

\section{Flow cytometry}

Synoviocytes and chondrocytes were seeded on plates for $12 \mathrm{~h}$. Next, the cells were treated with $0.25 \%$ trypsin for $24 \mathrm{~h}$ then harvested and washed twice with phosphate-buffered saline (PBS). The cell suspension was evaluated for apoptosis by double staining with annexin Vfluorescein isothiocyanate (FITC) and propidium iodide (PI) in binding buffer for $30 \mathrm{~min}$ at $37^{\circ} \mathrm{C}$. Apoptotic cells were detected using a FACS Calibur flow cytometer (BD Biosciences, CA). Flow cytometry analysis was repeated at least three times.

\section{Western blot analysis}

Isolated synoviocytes and chondrocytes were incubated at $37^{\circ} \mathrm{C}$ in $5 \% \mathrm{CO}_{2}$ for $24 \mathrm{~h}$ in $60-\mathrm{mm}$ dishes. Cells were then washed three times with PBS and were lyzed in 1X sodium dodecyl sulfate. Next, total protein extracts were subjected to sodium dodecyl sulfatepolyacrylamide gel electrophoresis and were transferred onto PVDF membranes. Thereafter, the membranes were blocked with $5 \%$ skim milk overnight at $4{ }^{\circ} \mathrm{C}$. After washing with TBS three times, the membranes were incubated with anticaspase- 3 and mouse monoclonal anti- $\beta$-actin (at a dilution of $1: 2000$ ) at $37^{\circ} \mathrm{C}$ for $1 \mathrm{~h}$. Next, the protein bands were developed with electrochemiluminescence reagents, and images were acquired using the ChemiDoc Imaging system. Western blot analysis was repeated at least three times.

\section{Statistical analysis}

Data are expressed as mean \pm standard deviation and were analyzed using one-way analysis of variance. Significance threshold was set at $p<0.05$. SPSS 17.0 was used for statistical analyses.

\section{RESULTS}

\section{Comparison of histopathological changes in $\mathrm{AA}$ and $\mathrm{CIA}$ rats}

To confirm the establishment of the rat model, the degree of foot swelling and histopathological changes of the knee joint were evaluated. As shown in Figure 1 ( $A$ and $B$ ), the secondary side of $A A$ and $\mathrm{CIA}$ rats showed obvious swelling and the score of the secondary arthritis index and degree of foot swelling were significantly higher than those of the normal group $(p<0.05)$, suggesting that the rat model was established.

After successful modeling, five rats were selected for each group, and blood was collected by retro-orbital venous plexus puncture. After sacrificing rats by cervical dislocation, the expression of Ptch1, Gli1, and Shh in articular chondrocytes and synovial cells were evaluated by Western blotting. Compared with the control group, the expression of Shh, Gli1, and Ptch1 protein in PBMCs, articular chondrocytes, and synoviocytes of $A A$ and collagen-induced $(\mathrm{CIA})$ rats were significantly increased $(p<0.05)$ (Figure $1 \mathrm{C}, \mathrm{D}$ and $\mathrm{E}$ ).

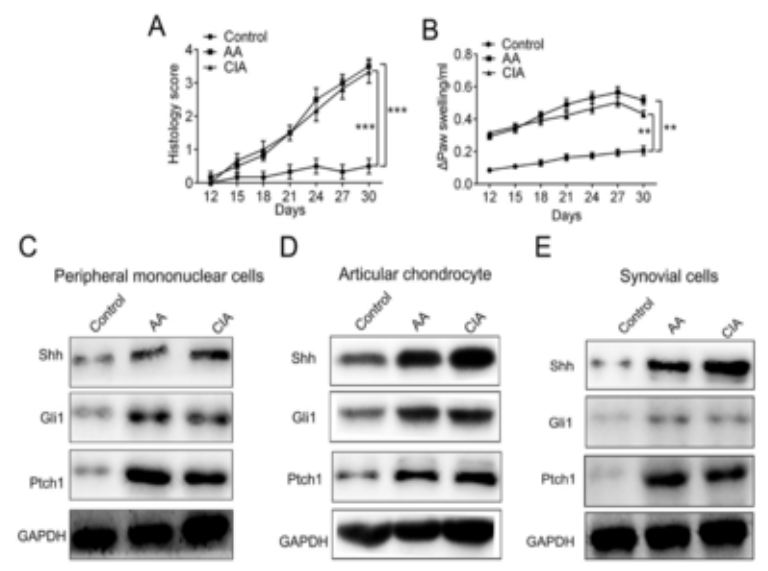

Figure 1: Comparison of histopathological changes in the knee joint $(A)$ and degree of foot swelling (B); Comparison of the expression of Shh, Gli1, and Ptch1 protein in peripheral mononuclear cells (C), chondrocytes (D) and synoviocytes (E) in normal rats (control group), CIA rats, and $\mathrm{AA}$ rats

\section{Effect of cyclopamine on proliferation and apoptosis of articular chondrocytes}

The effect of the $\mathrm{Hh}$ pathway inhibitor cyclopamine on the proliferation of articular chondrocytes is shown in Figure $2 \mathrm{~A}$. Cell proliferation rates of the $\mathrm{AA}$ and $\mathrm{CIA}$ groups were significantly higher than that of the control group $(p<0.05)$. In AA and ClA groups, $20 \mu \mathrm{mol} / \mathrm{L}$ of cyclopamine induced the highest level of cell proliferation $(1.24 \pm 0.11, p<0.05)$ and $(1.14 \pm$ $0.11, p<0.05)$, respectively. The cell proliferation increased by cyclopamine in a dosedependent manner $(p<0.05)$. Next, the effect of cyclopamine on apoptosis in articular chondrocytes was evaluated by Annexin VFITC/PI double staining. Following treatment with different concentrations of cyclopamine $(0.5,5$, 
and $20 \mu \mathrm{mol} / \mathrm{L}$ ), the percentages of apoptotic cells in the $A A$ and CIA groups were found to gradually decrease compared with that in the control group (Figure $2 \mathrm{~B}$ ). In the $\mathrm{AA}$ and $\mathrm{CIA}$ groups, $20 \mu \mathrm{mol} / \mathrm{L}$ of cyclopamine caused the lowest level of cell apoptosis $(0.24 \pm 0.03, p<$ $0.05)$ and $(0.17 \pm 0.02, p<0.05)$, respectively. Compared with the control group, the Shh, Gli1, and Ptch1 protein expression in cartilage cells of $A A$ and $C I A$ rats were significantly decreased ( $p$ $<0.05$ ) (Figure $2 \mathrm{C}$ ).

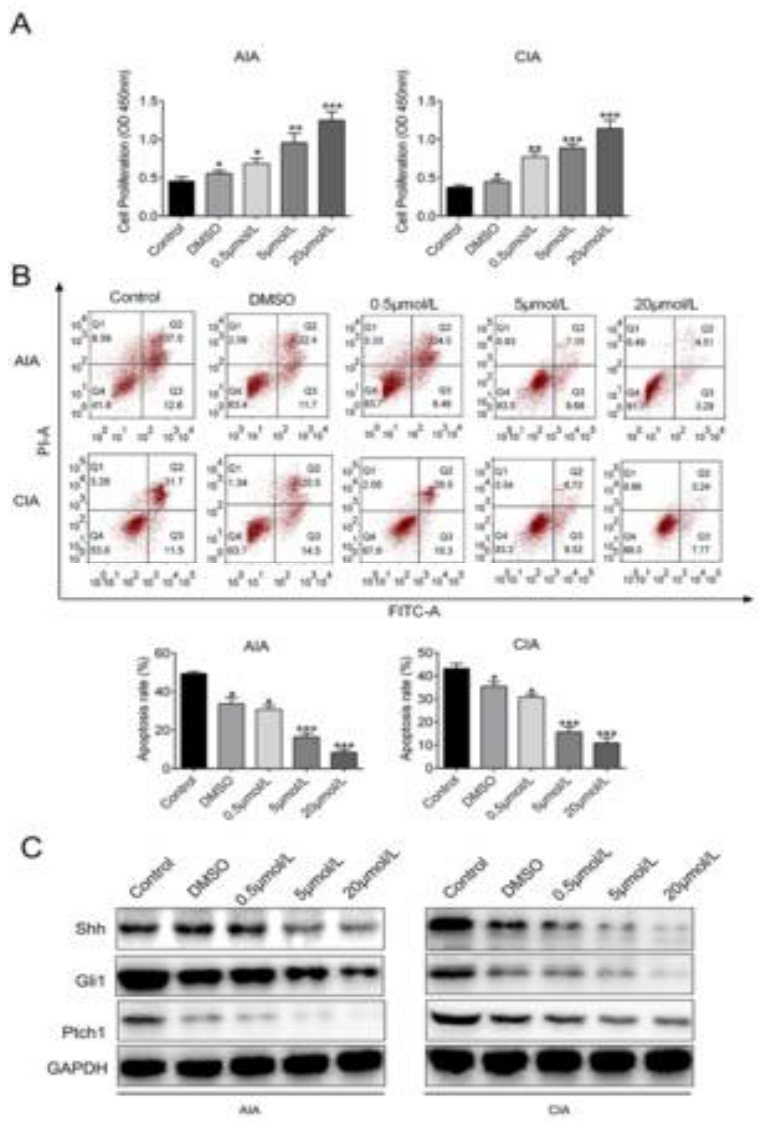

Figure 2: Effect of cyclopamine on the proliferation $(A)$ and apoptosis (B) of articular chondrocytes and $\mathrm{Hh}$ pathway-related protein expression $(C)$ in the cartilage cells of $A A$ rats and $C I A$ rats

\section{Effect of purmorphamine on proliferation of articular chondrocytes}

The effect of the $\mathrm{Hh}$ pathway activator purmorphamine on the proliferation articular chondrocytes is shown in Figure $3 \mathrm{~A}$. The cell proliferation rates of $\mathrm{AA}$ and $\mathrm{CIA}$ were significantly lower than that in the control group $(p<0.05)$. In the AA and CIA groups, the cell proliferation rate with $6 \mu \mathrm{mol} / \mathrm{L}$ of purmorphamine were the lowest $(8.31 \pm 1.74, p<0.05)$ and (10.54 $\pm 2.45, p<0.05)$, respectively. Cell proliferation rate was decreased with increase in the concentration of purmorphamine $(p<0.05)$. To quantitatively examine the effect of purmorphamine on apoptosis in articular chondrocytes, Annexin V-FITC/PI double staining was measured via flow cytometry. Following treatment with different concentrations of purmorphamine $(0.15,1.5,6 \mu \mathrm{mol} / \mathrm{L})$, the percentages of apoptotic cells of the AA and CIA groups were found to gradually increase when compared with that in the control group (Figure 3 B). In both AA and CIA groups, $6 \mu \mathrm{mol} / \mathrm{L}$ purmorphamine induced the highest level of cell apoptosis $[41.3 \pm 1.83(p<0.05)$ and $43.36 \pm$ $0.07(p<0.05)$, respectively]. Compared with the control group, the Shh, Gli1, and Ptch1 protein expression levels in the cartilage cells of AA and $\mathrm{CIA}$ rats were significantly increased $(p<0.05$, Figure $3 \mathrm{C}$ ).

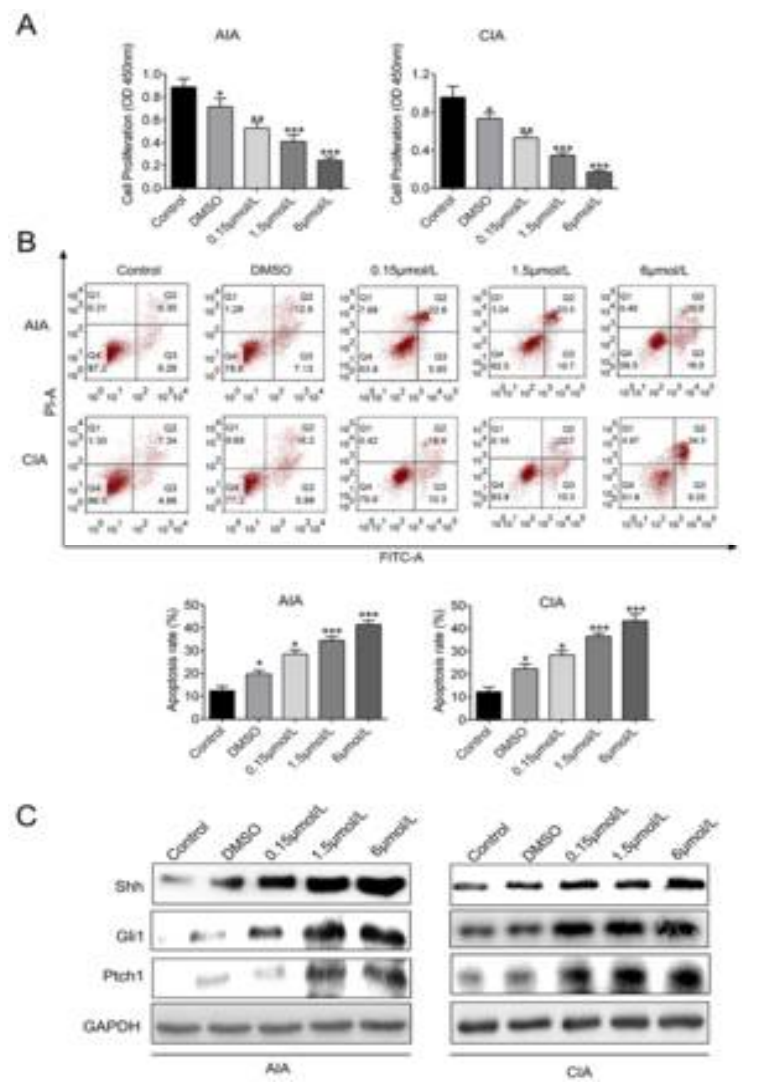

Figure 3: Effect of purmorphamine on the proliferation (A) and apoptosis (B) of articular chondrocytes and $\mathrm{Hh}$ pathway-related protein expression (C) in articular cartilage of $A A$ rats and $C I A$ rats

\section{DISCUSSION}

Rheumatoid arthritis is a chronic, and systemic inflammatory disease which is characterized by persistent inflammatory synovitis, affecting mainly the peripheral joints [18]. In China, the mortality rate of RA is $0.32-0.36 \%$, which is lower than the global average [19]. Wang et al. reported that in the absence of effective and timely treatment, more than $75 \%$ of patients with RA would be disabled within 3 years [20]. 
The Hh pathway plays an important role in the process of controlling cell migration, cell differentiation, and cell differentiation of embryonic cells [21]. It has been reported that the Hh pathway plays a complex role in the physiological and pathological processes of chondrocyte proliferation and differentiation, bone formation, and bone metabolism. In the present study, the Hh signal pathway was found to play a crucial role in the regulation of differentiation, development, and tumor formation in embryonic tissues.

AA and CIA models are typical RA models [22]. The pathological mechanisms of the $A A$ and $\mathrm{CIA}$ models are similar to that of human RA, but some differences exist between the models. The expression of the $\mathrm{Hh}$ signaling pathway in different RA models was investigated in the present study. Agonists and inhibitors were used to verify whether the $\mathrm{Hh}$ signaling pathway might play a role in the development of RA. In the present study, abnormal activation of the $\mathrm{Hh}$ signaling pathway in articular cartilage and synovial cells of $A A$ and CIA rats suggests that this pathway may be a new target for the treatment of RA. The Hh pathway inhibitor cyclopamine and activator purmorphamine can affect the protein expressions of Shh, Gli1, and Ptch1. Cyclopamine promoted the proliferation of chondrocytes and inhibited the excessive apoptosis of chondrocytes in vitro, suggesting that intervention of the $\mathrm{Hh}$ pathway in chondrocytes may be of potential therapeutic significance in protecting articular cartilage from RA.

\section{CONCLUSION}

This study, for the first time, reveals that $\mathrm{Hh}$ signal pathway is activated in the articular cartilage of AA and CIA rats. In vitro inhibition of $\mathrm{Hh}$ signaling by cyclopamine promote the cell proliferation rate and protein expression of Shh, Gli1, and Ptch1. However, cell apoptosis in AA and CIA groups are inhibited by cyclopamine. On the other hand, purmorphamine treatment causes the opposite effect. Thus, Hh signaling pathway contributes to the pathogenesis of cartilage destruction in RA. Further studies are needed to investigate the potential therapeutic effect of purmorphamine and cyclopamine, and their mechanisms of action in AA and CIA rats.

\section{DECLARATIONS}

\section{Conflict of Interest}

No conflict of interest associated with this work.

\section{Contribution of Authors}

We declare that this work was done by the authors named in this article and all liabilities pertaining to claims relating to the content of this article will be borne by the authors. Yingyi Wu designed all the experiments and revised the paper. Guangxia Yang and Jing Fei performed the experiments, Yang Huang wrote the paper and final approve.

\section{REFERENCES}

1. Salt E, Crofford L. Rheumatoid arthritis: new treatments, better outcomes. Nurse Pra 2012; 37(11): 22-23.

2. Araki $Y$, Mimura T. The Mechanisms Underlying Chronic Inflammation in Rheumatoid Arthritis from the Perspective of the Epigenetic Landscape. J Immunol Res. 2016; 2016(1): 629-682.

3. Keith MP, Edison JD, Gilliland WR. Progress Toward Personalized Treatment of Rheumatoid Arthritis. Clin Pharmacol Ther. 2012; 92(4): 440-442.

4. Day TF, Yang Y. Wnt and hedgehog signaling pathways in bone development. J Bone Joint Sur. 2008; 90(1): 1924.

5. Mak KK, Bi Y, Chao W, Chuang PT, Clemens T, Young $M$, Yang $Y$. Hedgehog signaling in mature osteoblasts regulates bone formation and resorption by controlling PTHrP and RANKL expression. Dev Cell. 2008; 14(5): 674-688.

6. Wilson CW, Chuang PT. Mechanism and evolution of cytosolic Hedgehog signal transduction. Development. 2010; 137(13): 2079.

7. Lin AC, Seeto BL, Bartoszko JM, Khoury MA, Whetstone $H$, Ho L, Hsu C, Ali SA, Alman BA. Modulating hedgehog signaling can attenuate the severity of osteoarthritis. Nature Med. 2009; 15(12): 1421-1425.

8. Wei F, Zhou J, Wei X, Zhang J, Fleming BC, Terek R, Pei $M$, Chen $Q$, Liu T, Wei L. Activation of Indian hedgehog promotes chondrocyte hypertrophy and upregulation of MMP-13 in human osteoarthritic cartilage. Osteoarthr Cartilage. 2012; 20(7): 755-763.

9. Buckland J. Osteoarthritis: blocking hedgehog signaling might have therapeutic potential in OA. Nature Rev Rheumatol. 2010; 6(2): 61.

10. Ali SA, Al-Jazrawe M, Ma $H$, Whetstone $H$, Poon $R$, Farr $S$, Naples $M$, Adeli $K$, Alman BA. Regulation of Cholesterol Homeostasis by Hedgehog Signaling in Osteoarthritic Cartilage. Arthrit Rheumatol. 2016; 68(1): 127-137.

11. Li R, Cai L, Ding J, Hu CM, Wu TN, Hu XY. Inhibition of hedgehog signal pathway by cyclopamine attenuates inflammation and articular cartilage damage in rats with adjuvant-induced arthritis. J Phar Pharmacol. 2015; 67(7): 963-971. 
12. Aug. International ethical guidelines for biomedical research involving human subjects. B Med Ethic. 2002; 10(182): 17-23.

13. Zeng JZ, Ma LF, Meng $H$, Yu HM, Zhang $Y K$, Guo A. (5R)-5-hydroxytriptolide (LLDT-8) prevents collageninduced arthritis through OPG/RANK/RANKL signaling in a rat model of rheumatoid arthritis. Exp Ther Med. 2016; 12(5): 3101-3107.

14. Ruknuddin G, Patgiri BJ, Prajapati PK, Ashok BK, Ravishankar B. Anti-arthritic Activity of Dashanga Ghana (An Ayurvedic Compound Formulation) Against Freund's Adjuvant Induced Arthritis in Charles Foster Albino Rats. Toxicol Int. 2015; 22(1): 141-148.

15. Chen XY, Li J, Cheng WM, Jiang H, Xie XF, Hu R. Effect of total flavonoids of Chrysanthemum indicum on the apoptosis of synoviocytes in joint of adjuvant arthritis rats. Am J Chinese Med. 2008; 36(04): 695-704.

16. Chen $Q Q$, Xiao $P$, Liu $H X$, Zhang YF, Stomatology $D O$. Cultivation of condylar chondrocytes and its biological characteristics in rats. J Chinese Prac Diag Ther. 2017.

17. Iwamoto $T$, Okamoto $H$, Shu K, Ikari K, Toyama $Y$, Tomatsu T, Kamatani N, Momohara S. A role of monocyte chemoattractant protein-4 (MCP-4)/CCL13 from chondrocytes in rheumatoid arthritis. Febs J. 2010; 274(18): 4904-4912.
18. Kanbe K, Oh K, Chiba J, Inoue Y, Taguchi M, Yabuki A. Analysis of Mitogen-Activated Protein Kinases in Bone and Cartilage of Patients with Rheumatoid Arthritis Treated with Abatacept. Clin Med Insigh Arthr Musculoskeletal Disord. 2016; 9(9): 51-56.

19. Chen XX, Dai $Q$, Bao $C D$, Wu HX, Zhao DB. $A$ multicenter, randomized, double-blind clinical trial of combination therapy with Anbainuo, a novel recombinant human TNFRII:FC fusion protein, plus methotrexate versus methotrexate alone or Anbainuo alone in Chinese patients with moderate to severe rheum. Clin Rheumatol. 2013; 32(1): 99-108.

20. Wang $H$, Chen W, Wang L, Li F, Zhang C, XU L. Tumor necrosis factor receptor-associated factor 6 promotes migration of rheumatoid arthritis fibroblast-like synoviocytes. Mol Med Rep. 2015; 11(4): 2761-2766.

21. Ingham PW, Mcmahon AP. Hedgehog signaling in animal development: paradigms and principles. Gene Dev. 2001; 15(15): 3059-3087.

22. Milici AJ, Kudlacz EM, Audoly L, Zwillich S, Changelian $P$. Cartilage preservation by inhibition of Janus kinase 3 in two rodent models of rheumatoid arthritis. Arthritis Res Ther 2008; 10(1): R14-R14. 